\title{
A Cascaded Reduced-Dimension STAP Method for Airborne MIMO Radar in the Presence of Jammers
}

\author{
Ting WANG, Yongjun ZHAO, Shiwen CHEN, Kunfan ZHANG \\ Zhengzhou Institute of Information Science and Technology, Science Road 62, Zhengzhou, People's Republic of China \\ wangtingsp@163.com, zhaoyjzz@163.com,ndsccsw@126.com,my_chocolate@163.com
}

Submitted March 11, 2016 / Accepted January 2, 2017

\begin{abstract}
A cascaded reduced-dimension (RD) space-time adaptive processing (STAP) method for airborne multipleinput multiple-output (MIMO) radar in the presence of jammers is proposed in this paper. The proposed MIMOSTAP method for clutter plus jamming suppression proceeds in two steps. Firstly, the jamming is suppressed by its orthogonal complementary subspace obtained in the passive radar mode, while the receive dimension is reduced. Secondly, the tri-iterative algorithm (TRIA) is utilized to suppress the clutter combining the remaining receive degree of freedom (DOF) with the transmit DOF and the Doppler DOF, and further dimension reduction is implemented. The proposed method can effectively realize the separate jamming and clutter elimination. Moreover, the training sample number and the computational complexity are significantly decreased. Simulation results verify the validity of the proposed cascaded RD MIMO-STAP method under jamming condition.
\end{abstract}

\section{Keywords}

Multiple-input multiple-output (MIMO) radar, spacetime adaptive processing (STAP), dimension reduction, jammer, tri-iterative algorithm (TRIA)

\section{Introduction}

Multiple-input multiple-output (MIMO) radar has drawn considerable attention in recent years [1-5]. According to the system configuration, two classes of MIMO radar are mainly investigated, i.e., distributed MIMO radar [2], [3] and collocated MIMO radar [4]. Because of the waveform diversity property, the collocated MIMO radar can produce extended virtual array aperture and enlarge the system degree of freedom (DOF) [4], [5]. Thus the collocated MIMO radar is applicable for the airborne platform which severely limits the aperture length and the weight of the airborne radar [5], [6], [7]. Meanwhile, space-time adaptive processing (STAP) [8], [9], [10] has become a crucial technique for the airborne radar to suppress the clutter and realize the ground moving target indication (GMTI). Nowadays, the airborne MIMO radar is combined with STAP technique to improve the clutter suppression and GMTI performance [11-15].

Due to the extra DOF provided by the waveform diversity, MIMO-STAP system enlarges the requirement of the independent and identically distributed (IID) training samples according to the well-known Reed-Mallett-Brennan (RMB) rule [16], and suffers a tremendous increase in computational cost. These practical problems have severely limited the development and application of MIMO-STAP. Up to now, various reduced-dimension (RD) STAP algorithms [12-15] have been developed for airborne MIMO radar to decrease the training sample requirement and the computational burden, such as the classical tri-iterative algorithm (TRIA) [14], [15].

Moreover, the practical operation environment of airborne radar becomes progressively complicated, and the target detection capability of airborne radar is usually affected by jamming and other interferences [10], [17], [18]. The existence of jammers will further bring additional processing difficulty to the conventional STAP system [10]. Therefore, we should investigate effective STAP approaches in the presence of jammers to mitigate the clutter plus jamming. To the best of our knowledge, the state-of-art research focusing on the MIMO-STAP method under jamming condition is still relatively less.

Aiming at the above problem and the research status, a cascaded RD MIMO-STAP method is proposed in this paper. Two stages are involved in this method. In the first stage, the orthogonal complementary subspace of jamming is obtained in the passive radar mode, and partial receive DOF is utilized to cancel the jamming. In the second stage, we conduct the TRIA to suppress the clutter after the matched-filtering and time-delaying, and to further reduce the dimension. Therefore, the separate jammer and clutter rejection is performed, and the computational cost is efficiently reduced as well as the required IID sample number. Simulation results are provided to verify the effectiveness of the proposed MIMO-STAP method for clutter plus jamming suppression.

The rest of the paper is organized as follows. The signal model of airborne MIMO radar STAP is established in Sec. 2, including the jamming and clutter signals. Then 
Section 3 illustrates the principle of the proposed cascaded RD MIMO-STAP method according to two steps, and analyzes the sample requirement and the computational complexity. The simulation results are presented in Sec. 4 to demonstrate the performance of the proposed method. Finally, we draw the conclusion in Sec. 5.

Notations. The operators $(\cdot)^{\mathrm{T}},(\cdot)^{\mathrm{H}},(\cdot)^{-1}, E[\cdot]$ and $\otimes$ denote the transpose, conjugate transpose, inverse, expectation and Kronecker product, respectively. $\delta(\cdot)$ stands for the unit impulse function. The symbol $\operatorname{diag}\left\{r_{1}, r_{2}\right\}$ represents a diagonal matrix with diagonal entries $r_{1}, r_{2} . \mathbf{I}_{M}$ denotes the $M \times M$ identity matrix.

\section{Signal Model of Airborne MIMO Radar STAP}

Figure 1 presents a side-looking monostatic MIMO radar system equipped with collocated transmit and receive linear arrays, in which there are $M$ transmit elements with uniform spacing $d_{\mathrm{t}}$ and $N$ receive elements with uniform spacing $d_{\mathrm{r}}$, and sparse coefficient $\alpha=d_{\mathrm{t}} / d_{\mathrm{r}}$, where $d_{\mathrm{r}}=\lambda / 2$ and $\lambda$ is the wavelength. A coherent processing interval (CPI) consists of $K$ pulses with a constant radar pulse repetition interval (PRI) $T$. The radar platform travels at height $H$ and at velocity $V$. The cone angle between the line-of-sight (LOS) and the velocity vector is $\psi$, while $\theta$ and $\varphi$ are the azimuth angle and the elevation angle, respectively. The number of clutter patches which are uniformly distributed in a range cell is $N_{\mathrm{c}}$. The clutter data vector of MIMO-STAP can be expressed as

$$
\begin{aligned}
\mathbf{y}_{\mathrm{c}} & =\sum_{i=1}^{N_{\mathrm{c}}} \rho_{i} \mathbf{v}\left(f_{\mathrm{sc}, i}, f_{\mathrm{dc}, i}\right) \\
& =\sum_{i=1}^{N_{\mathrm{c}}} \rho_{i} \mathbf{a}_{\mathrm{d}}\left(f_{\mathrm{dc}, i}\right) \otimes \mathbf{a}_{\mathrm{t}}\left(f_{\mathrm{sc}, i}\right) \otimes \mathbf{a}_{\mathrm{r}}\left(f_{\mathrm{sc}, i}\right)
\end{aligned}
$$

where $\rho_{i}$ is the reflect coefficient of the clutter patch at $\theta_{i}$, $\mathbf{v}\left(f_{\mathrm{sc}, i}, f_{\mathrm{dc}, i}\right)=\mathbf{a}_{\mathrm{d}}\left(f_{\mathrm{dc}, i}\right) \otimes \mathbf{a}_{\mathrm{t}}\left(f_{\mathrm{sc}, i}\right) \otimes \mathbf{a}_{\mathrm{r}}\left(f_{\mathrm{sc}, i}\right)$ is the whole space-time steering vector of MIMO-STAP for the clutter patch at $\theta_{i}, \mathbf{a}_{\mathrm{d}}\left(f_{\mathrm{dc}, i}\right)=\left[\begin{array}{llll}1 & \exp \left(\mathrm{j} 2 \pi f_{\mathrm{dc}, i}\right) & \cdots & \exp \left(\mathrm{j} 2 \pi(K-1) f_{\mathrm{dc}, i}\right)\end{array}\right]^{\mathrm{T}}$ $=\left[\begin{array}{llll}1 & \exp \left(\mathrm{j} 2 \pi \beta f_{\mathrm{sc}, i}\right) & \cdots & \exp \left(\mathrm{j} 2 \pi(K-1) \beta f_{\mathrm{sc}, i}\right)\end{array}\right]^{\mathrm{T}}$ $\mathbf{a}_{\mathrm{t}}\left(f_{\mathrm{sc}, i}\right)=\left[\begin{array}{llll}1 & \exp \left(\mathrm{j} 2 \pi \alpha f_{\mathrm{sc}, i}\right) & \cdots & \exp \left(\mathrm{j} 2 \pi(M-1) \alpha f_{\mathrm{sc}, i}\right.\end{array}\right]^{\mathrm{T}} \quad$ and $\mathbf{a}_{\mathrm{r}}\left(f_{\mathrm{sc}, i}\right)=\left[\begin{array}{llll}1 & \exp \left(\mathrm{j} 2 \pi f_{\mathrm{sc}, i}\right) & \cdots & \exp \left(\mathrm{j} 2 \pi(N-1) f_{\mathrm{sc}, i}\right)\end{array}\right]^{\mathrm{T}}$ are steering vectors of temporal Doppler, spatial transmit array, and spatial receive array, respectively, $f_{\mathrm{dc}, i}=2 V T \cos \psi_{i} / \lambda$ $=2 V T \cos \theta_{i} \cos \varphi / \lambda$ and $f_{\mathrm{sc}, i}=d_{\mathrm{r}} \cos \psi_{i} / \lambda=d_{\mathrm{r}} \cos \theta_{i} \cos \varphi / \lambda$ are the normalized Doppler and spatial frequencies of the clutter patch at $\theta_{i}$, respectively, $\beta=f_{\mathrm{dc}, i} / f_{\mathrm{sc}, i}=2 V T / d_{\mathrm{r}}$ and $i=1,2, \cdots, N_{\mathrm{c}}$.

The clutter covariance matrix can be expressed as

$$
\mathbf{R}_{\mathrm{C}}=E\left[\mathbf{y}_{\mathrm{c}} \mathbf{y}_{\mathrm{c}}^{\mathrm{H}}\right]=\sum_{i=1}^{N_{\mathrm{c}}} \sigma_{\mathrm{c}, i}^{2} \mathbf{v}\left(f_{\mathrm{sc}, i}, f_{\mathrm{dc}, i}\right) \mathbf{v}^{\mathrm{H}}\left(f_{\mathrm{sc}, i}, f_{\mathrm{dc}, i}\right)
$$

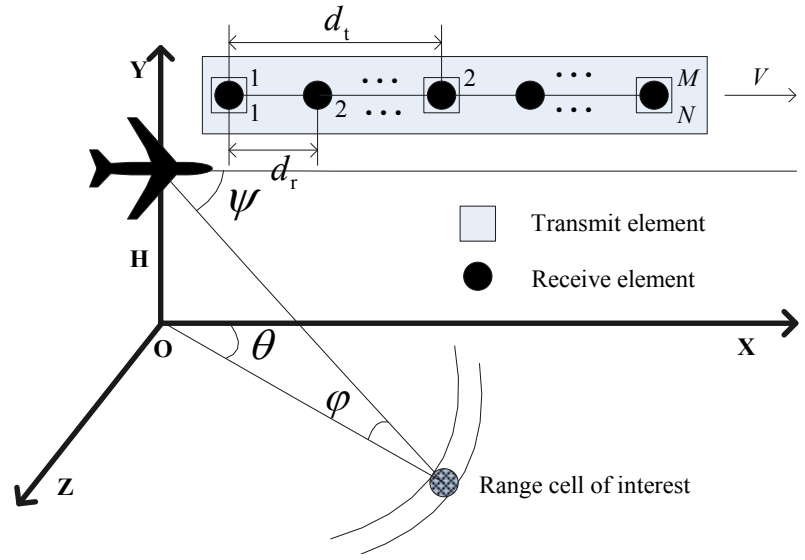

Fig. 1. Configuration of airborne MIMO radar.

where $\sigma_{\mathrm{c}, i}^{2}$ is the variance of the $i$ th clutter patch, which is in direct proportion to the radar cross section (RCS) and satisfies $E\left[\left|\rho_{i} \rho_{k}^{*}\right|\right]=\sigma_{\mathrm{c}, i}^{2} \delta(i-k), i, k=1,2, \cdots, N_{\mathrm{c}}$.

Assume there exist $J$ independent jammers in practical circumstance. And each jammer is independent at different times in temporal domain, i.e., it is Doppler white. Then the jamming covariance matrix can be written as

$$
\mathbf{R}_{\mathrm{J}}=\mathbf{I}_{K M} \otimes \mathbf{R}_{\mathrm{rJ}}=\mathbf{I}_{K M} \otimes\left[\sum_{i=1}^{J} \sigma_{\mathrm{j}, i}^{2} \mathbf{a}_{\mathrm{r}}\left(f_{\mathrm{sj}, i}\right) \mathbf{a}_{\mathrm{r}}^{\mathrm{H}}\left(f_{\mathrm{s} \mathrm{j}, i}\right)\right]
$$

where $\sigma_{\mathrm{j}, i}^{2}$ is the variance of the $i$ th jammer, and $f_{\mathrm{s}, i}$ is the normalized spatial frequency of the $i$ th jammer.

Assume the noise obeys white Gaussian distribution in both the spatial and temporal domains, thus the noise covariance matrix can be written as

$$
\mathbf{R}_{\mathrm{W}}=\sigma_{\mathrm{w}}^{2} \mathbf{I}_{K M N}
$$

where $\sigma_{\mathrm{w}}^{2}$ is the variance of the additive white noise.

Considering the clutter, the jammer and the noise exist at the same time, the fully-adaptive weight vector $\mathbf{w} \in \mathbb{C}^{K M N \times 1}$ for MIMO radar STAP can be calculated by

$$
\mathbf{w}=\frac{\mathbf{R}^{-1} \mathbf{v}\left(f_{\mathrm{s}, 0}, f_{\mathrm{d}, 0}\right)}{\mathbf{v}^{\mathrm{H}}\left(f_{\mathrm{s}, 0}, f_{\mathrm{d}, 0}\right) \mathbf{R}^{-1} \mathbf{v}\left(f_{\mathrm{s}, 0}, f_{\mathrm{d}, 0}\right)}
$$

where $\mathbf{R}=\mathbf{R}_{\mathrm{C}}+\mathbf{R}_{\mathrm{J}}+\mathbf{R}_{\mathrm{W}}$ represents the clutter-plus-jamming-plus-noise covariance matrix, and $\mathbf{v}\left(f_{\mathrm{s}, 0}, f_{\mathrm{d}, 0}\right)=$ $=\mathbf{a}_{\mathrm{d}}\left(f_{\mathrm{d}, 0}\right) \otimes \mathbf{a}_{\mathrm{t}}\left(f_{\mathrm{s}, 0}\right) \otimes \mathbf{a}_{\mathrm{r}}\left(f_{\mathrm{s}, 0}\right)$ is the space-time steering vector of target, $f_{\mathrm{s}, 0}$ and $f_{\mathrm{d}, 0}$ are the normalized spatial and Doppler frequencies of the target.

The full-dimension optimum STAP can suppress the clutter and jamming simultaneously. With the extension of processing dimension in MIMO radar, the training sample number $L$ of the full-dimension MIMO-STAP should meet the requirement $L \geq 2 K M N$ to estimate the matrix $\mathbf{R}$ efficiently [16]. However, it is very difficult to acquire enough IID samples in the practical non-homogeneous clutter scenarios. Moreover, the complexity to compute the matrix 
estimation and inversion is $\mathrm{O}\left(L K^{2} M^{2} N^{2}\right)+\mathrm{O}\left(K^{3} M^{3} N^{3}\right)$. The problem of computational cost is so challenging that the real-time processing can hardly be realized [8], [9], [10]. Therefore, the effective RD techniques should be investigated for MIMO-STAP under jamming condition.

\section{Cascaded RD MIMO-STAP Method for Clutter plus Jamming Suppres- sion}

Exploiting the different signal model characteristics between jamming and clutter, we put forward a cascaded RD MIMO-STAP method in this section. The proposed method is conducted in two steps: (1) suppressing the jamming by its orthogonal complementary subspace; (2) suppressing the clutter by utilizing TRIA. The processing diagram is illustrated in Fig. 2.

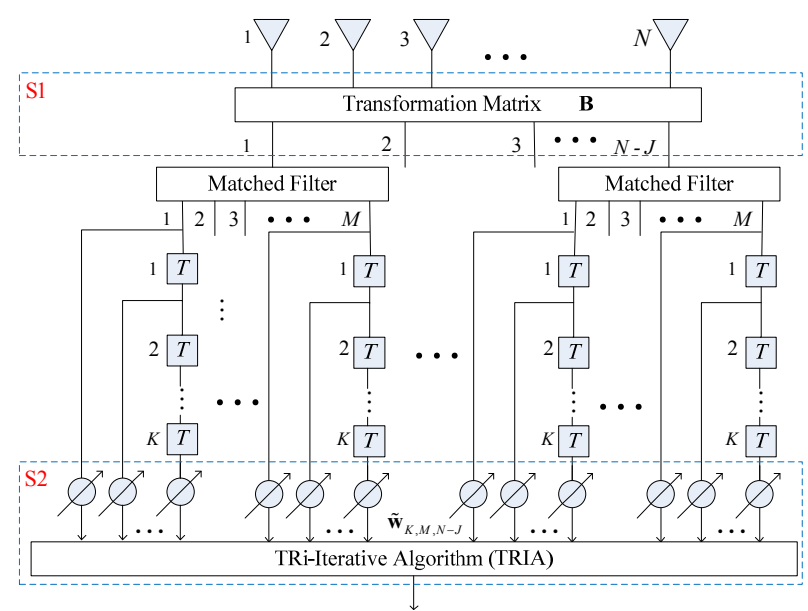

Fig. 2. Processing diagram of the proposed method.

\subsection{Jamming Cancellation Based on Subspace Orthogonality}

In the first stage, we utilize a transformation matrix B to suppress the jamming in the spatial receive domain, which is determined by the $N \times 1$ receive signals of the first pulse in passive radar mode. When the airborne MIMO radar operates in the passive mode, only the jamming and the noise are acquired at the receive site, while the target signal and the clutter are not contained.

Then the receive jamming plus noise covariance matrix in passive mode can be described as

$$
\mathbf{R}_{\mathrm{r}}=\sum_{i=1}^{J} \sigma_{\mathrm{j}, i}^{2} \mathbf{a}_{\mathrm{r}}\left(f_{\mathrm{sj}, i}\right) \mathbf{a}_{\mathrm{r}}^{\mathrm{H}}\left(f_{\mathrm{sj}, i}\right)+\mathbf{I}_{N} .
$$

Through performing eigenvalue decomposition (EVD) on $\mathbf{R}_{\mathrm{r}}$, we can obtain

$$
\mathbf{R}_{\mathrm{r}}=\mathbf{E}_{\mathrm{J}} \boldsymbol{\Lambda}_{1} \mathbf{E}_{\mathrm{J}}^{\mathrm{H}}+\mathbf{E}_{\mathrm{J}}^{\perp} \boldsymbol{\Lambda}_{2} \mathbf{E}_{\mathrm{J}}^{\perp \mathrm{H}}
$$

where $\boldsymbol{\Lambda}_{1}=\operatorname{diag}\left(\lambda_{1}, \lambda_{2}, \cdots \lambda_{J}\right), \boldsymbol{\Lambda}_{2}=\operatorname{diag}\left(\lambda_{J+1}, \lambda_{J+2}, \cdots \lambda_{N}\right)$, $\lambda_{1} \geq \lambda_{2} \geq \cdots \geq \lambda_{J} \geq \lambda_{J+1}=\lambda_{J+2}=\cdots=\lambda_{N}, \quad \mathbf{E}_{\mathrm{J}} \in \mathbb{C}^{N \times J} \quad$ and $\mathbf{E}_{\mathrm{J}}^{\perp} \in \mathbb{C}^{N \times(N-J)}$ are the matrix of eigenvectors corresponding to $\boldsymbol{\Lambda}_{1}$ and $\boldsymbol{\Lambda}_{2}$, respectively. Based on the subspace property in matrix theory [19], $\mathbf{E}_{\mathrm{J}}$ constitutes the jamming subspace, while $\mathbf{E}_{\mathrm{J}}^{\perp}$ constitutes the orthogonal complementary subspace of jamming. Then we select $\mathbf{B}=\mathbf{E}_{\mathrm{J}}^{\perp}$ as the transformation matrix to function on the radar echo, which is orthogonal to the jamming subspace. Thus, the jamming signal is suppressed when MIMO radar operates in active mode, and the dimension reduction is implemented in the spatial receive domain at the same time (from $N$ to $N-J$ ).

\subsection{Clutter Rejection Based on TRIA}

After the jamming suppression stage in active radar mode, the $k$ th echo pulse of the clutter signal received by $(N-J)$ channels can be written as

$$
\tilde{\mathbf{x}}_{\mathrm{c}}(t, k)=\sum_{i=1}^{N_{\mathrm{c}}} \rho_{i} \exp \left(\mathrm{j} 2 \pi(k-1) f_{\mathrm{dc}, i}\right) \mathbf{B}^{\mathrm{H}} \mathbf{a}_{\mathrm{r}}\left(f_{\mathrm{sc}, i}\right) \mathbf{a}_{\mathrm{t}}^{\mathrm{T}}\left(f_{\mathrm{sc}, i}\right) \mathbf{s}(t)
$$

where $\mathbf{s}(t)$ represents the transmit waveform vector of MIMO radar which satisfies the orthogonality condition

$$
\int_{T_{0}} \mathbf{s}(t) \mathbf{s}^{\mathrm{H}}(t)=\mathbf{I}_{M} .
$$

where $T_{0}$ is the pulse width.

Next, the clutter output of the matched filters with $M$ transmit waveforms behind each channel is expressed as

$$
\begin{aligned}
\tilde{\mathbf{X}}_{\mathrm{c}}(k) & =\int_{T_{0}} \tilde{\mathbf{x}}_{\mathrm{c}}(t, k) \mathbf{s}^{\mathrm{H}}(t) \mathrm{d} t \\
& =\sum_{i=1}^{N_{\mathrm{c}}} \rho_{i} \exp \left(\mathrm{j} 2 \pi(k-1) f_{\mathrm{dc}, i}\right) \mathbf{B}^{\mathrm{H}} \mathbf{a}_{\mathrm{r}}\left(f_{\mathrm{sc}, i}\right) \mathbf{a}_{\mathrm{t}}^{\mathrm{T}}\left(f_{\mathrm{sc}, i}\right) .
\end{aligned}
$$

Further, by stacking the $K$ pulse data $\tilde{\mathbf{Y}}_{\mathrm{c}}=\left[\begin{array}{llll}\tilde{\mathbf{X}}_{\mathrm{c}}(1) & \tilde{\mathbf{X}}_{\mathrm{c}}(2) & \cdots & \tilde{\mathbf{X}}_{\mathrm{c}}(K)\end{array}\right]$ of the delay lines according to the column order, we can obtain the space-time data vector of the clutter signal after jamming suppression

$$
\tilde{\mathbf{y}}_{\mathrm{c}}=\sum_{i=1}^{N_{\mathrm{c}}} \rho_{i} \mathbf{a}_{\mathrm{d}}\left(f_{\mathrm{dc}, i}\right) \otimes \mathbf{a}_{\mathrm{t}}\left(f_{\mathrm{sc}, i}\right) \otimes\left[\mathbf{B}^{\mathrm{H}} \mathbf{a}_{\mathrm{r}}\left(f_{\mathrm{sc}, i}\right)\right] .
$$

Comparing (11) to (1), we can find that the spatial receive dimension of the MIMO-STAP signal model has been reduced from $N$ to $N-J$, as a consequence of the transformation matrix $\mathbf{B}$ in the first stage.

Meanwhile, the clutter covariance matrix can be written as

$$
\begin{aligned}
\tilde{\mathbf{R}}_{\mathrm{C}}= & E\left[\tilde{\mathbf{y}}_{\mathrm{c}} \tilde{\mathbf{y}}_{\mathrm{c}}^{\mathrm{H}}\right] \\
= & \sum_{i=1}^{N_{\mathrm{c}}} \sigma_{\mathrm{c}, i}^{2}\left[\mathbf{a}_{\mathrm{d}}\left(f_{\mathrm{dc}, i}\right) \mathbf{a}_{\mathrm{d}}^{\mathrm{H}}\left(f_{\mathrm{dc}, i}\right)\right] \otimes\left[\mathbf{a}_{\mathrm{t}}\left(f_{\mathrm{sc}, i}\right) \mathbf{a}_{\mathrm{t}}^{\mathrm{H}}\left(f_{\mathrm{sc}, i}\right)\right] \\
& \otimes\left[\mathbf{B}^{\mathrm{H}} \mathbf{a}_{\mathrm{r}}\left(f_{\mathrm{sc}, i}\right) \mathbf{a}_{\mathrm{r}}^{\mathrm{H}}\left(f_{\mathrm{sc}, i}\right) \mathbf{B}\right] .
\end{aligned}
$$


Then after the similar corresponding processing, the jamming covariance matrix and the noise covariance matrix can be respectively expressed as

$$
\begin{aligned}
& \tilde{\mathbf{R}}_{\mathrm{J}}=\sum_{i=1}^{J} \sigma_{\mathrm{j}, i}^{2} \mathbf{I}_{K M} \otimes\left[\mathbf{B}^{\mathrm{H}} \mathbf{a}_{\mathrm{r}}\left(f_{\mathrm{s}, i, i}\right) \mathbf{a}_{\mathrm{r}}^{\mathrm{H}}\left(f_{\mathrm{sj}, i}\right) \mathbf{B}\right], \\
& \tilde{\mathbf{R}}_{\mathrm{W}}=\sigma_{\mathrm{w}}^{2} \mathbf{I}_{K M(N-J)} \otimes\left[\mathbf{B}^{\mathrm{H}} \mathbf{B}\right]=\sigma_{\mathrm{w}}^{2} \mathbf{I}_{K M(N-J)} .
\end{aligned}
$$

Due to the orthogonality between $\mathbf{B}$ and the jamming subspace [19], we can come to the conclusion that the jamming covariance matrix $\tilde{\mathbf{R}}_{\mathrm{J}}=\mathbf{0}$ in theory. We define $\tilde{\mathbf{R}}=$ $=\tilde{\mathbf{R}}_{\mathrm{C}}+\tilde{\mathbf{R}}_{\mathrm{W}}$ and $\tilde{\mathbf{v}}\left(f_{\mathrm{s}, 0}, f_{\mathrm{d}, 0}\right)=\mathbf{a}_{\mathrm{d}}\left(f_{\mathrm{d}, 0}\right) \otimes \mathbf{a}_{\mathrm{t}}\left(f_{\mathrm{s}, 0}\right) \otimes\left[\mathbf{B}^{\mathrm{H}} \mathbf{a}_{\mathrm{r}}\left(f_{\mathrm{s}, 0}\right)\right]$, then the clutter suppression can be expressed as the following optimization problem

$$
\begin{aligned}
& \min _{\tilde{\mathbf{w}}} \tilde{\mathbf{w}}^{\mathrm{H}} \tilde{\mathbf{R}} \tilde{\mathbf{w}} \\
& \text { subject to } \tilde{\mathbf{w}}^{\mathrm{H}} \tilde{\mathbf{v}}\left(f_{\mathrm{s}, 0}, f_{\mathrm{d}, 0}\right)=1 .
\end{aligned}
$$

Then based on the idea of TRIA [14], [15] and the structure of the novel clutter model, the whole weight vector $\tilde{\mathbf{w}} \in \mathbb{C}^{K M(N-J) \times 1}$ can be described as the following separable form

$$
\tilde{\mathbf{w}}=\tilde{\mathbf{w}}_{\mathrm{d}} \otimes \tilde{\mathbf{w}}_{\mathrm{t}} \otimes \tilde{\mathbf{w}}_{\mathrm{r}}
$$

where $\tilde{\mathbf{w}}_{\mathrm{d}} \in \mathbb{C}^{K \times 1}, \quad \tilde{\mathbf{w}}_{\mathrm{t}} \in \mathbb{C}^{M \times 1}$ and $\tilde{\mathbf{w}}_{\mathrm{r}} \in \mathbb{C}^{(N-J) \times 1}$ are the reduced-dimension weight vectors corresponding to the Doppler, the transmit and the receive parts, respectively.

Thus (15) can be further rewritten as

$$
\begin{aligned}
& \min _{\tilde{\mathbf{w}}_{\mathrm{d}}, \tilde{\mathbf{w}}_{\mathrm{t}}, \tilde{\mathbf{w}}_{\mathrm{r}}}\left(\tilde{\mathbf{w}}_{\mathrm{d}} \otimes \tilde{\mathbf{w}}_{\mathrm{t}} \otimes \tilde{\mathbf{w}}_{\mathrm{r}}\right)^{\mathrm{H}} \tilde{\mathbf{R}}\left(\tilde{\mathbf{w}}_{\mathrm{d}} \otimes \tilde{\mathbf{w}}_{\mathrm{t}} \otimes \tilde{\mathbf{w}}_{\mathrm{r}}\right) \\
& \text { subject to }\left(\tilde{\mathbf{w}}_{\mathrm{d}} \otimes \tilde{\mathbf{w}}_{\mathrm{t}} \otimes \tilde{\mathbf{w}}_{\mathrm{r}}\right)^{\mathrm{H}} \tilde{\mathbf{v}}\left(f_{\mathrm{s}, 0}, f_{\mathrm{d}, 0}\right)=1 .
\end{aligned}
$$

According to the above formulation, the implementation steps to determine the low-dimension adaptive weight vectors $\tilde{\mathbf{w}}_{\mathrm{d}}, \tilde{\mathbf{w}}_{\mathrm{t}}$ and $\tilde{\mathbf{w}}_{\mathrm{r}}$ through solving (17) are summarized as follows:

(a) Given the normalized initial values $\tilde{\mathbf{w}}_{\mathrm{d}}(0)=\mathbf{a}_{\mathrm{d}}\left(f_{\mathrm{d}, 0}\right) /\left\|\mathbf{a}_{\mathrm{d}}\left(f_{\mathrm{d}, 0}\right)\right\|$ and $\tilde{\mathbf{w}}_{\mathrm{t}}(0)=\mathbf{a}_{\mathrm{t}}\left(f_{\mathrm{s}, 0}\right) /\left\|\mathbf{a}_{\mathrm{t}}\left(f_{\mathrm{s}, 0}\right)\right\| ;$

(b) Construct the receive reduced-dimension transformation matrix $\mathbf{T}_{\mathrm{r}}=\tilde{\mathbf{w}}_{\mathrm{d}}(i-1) \otimes \tilde{\mathbf{w}}_{\mathrm{t}}(i-1) \otimes \mathbf{I}_{N-J} \in \mathbb{C}^{K M(N-J) \times(N-J)} \quad, \quad$ and derive the receive reduced-dimension covariance matrix $\tilde{\mathbf{R}}_{\mathrm{r}}=\mathbf{T}_{\mathrm{r}}^{\mathrm{H}} \tilde{\mathbf{R}} \mathbf{T}_{\mathrm{r}}$ and the corresponding receive steering vector $\tilde{\mathbf{v}}_{\mathrm{r}}=\mathbf{T}_{\mathrm{r}}^{\mathrm{H}} \tilde{\mathbf{v}}\left(f_{\mathrm{s}, 0}, f_{\mathrm{d}, 0}\right)$. Then calculate the receive weight vector $\tilde{\mathbf{w}}_{\mathrm{r}}(i)=\tilde{\mathbf{R}}_{\mathrm{r}}^{-1} \tilde{\mathbf{v}}_{\mathrm{r}} / \tilde{\mathbf{v}}_{\mathrm{r}}^{\mathrm{H}} \tilde{\mathbf{R}}_{\mathrm{r}}^{-1} \tilde{\mathbf{v}}_{\mathrm{r}}$, where $i=1,2, \cdots$ is the iteration number;

(c) Construct the transmit reduced-dimension transformation matrix $\mathbf{T}_{\mathrm{t}}=\tilde{\mathbf{w}}_{\mathrm{d}}(i-1) \otimes \mathbf{I}_{M} \otimes \tilde{\mathbf{w}}_{\mathrm{r}}(i) \in \mathbb{C}^{K M(N-J) \times M}$, and derive the transmit reduced-dimension covariance matrix $\tilde{\mathbf{R}}_{\mathrm{t}}=\mathbf{T}_{\mathrm{t}}^{\mathrm{H}} \tilde{\mathbf{R}} \mathbf{T}_{\mathrm{t}}$ and the corresponding transmit steering vector $\tilde{\mathbf{v}}_{\mathrm{t}}=\mathbf{T}_{\mathrm{t}}^{\mathrm{H}} \tilde{\mathbf{v}}\left(f_{\mathrm{s}, 0}, f_{\mathrm{d}, 0}\right)$. Then calculate the transmit weight vector $\tilde{\mathbf{w}}_{\mathrm{t}}(i)=\tilde{\mathbf{R}}_{\mathrm{t}}^{-1} \tilde{\mathbf{v}}_{\mathrm{t}} / \tilde{\mathbf{v}}_{\mathrm{t}}^{\mathrm{H}} \tilde{\mathbf{R}}_{\mathrm{t}}^{-1} \tilde{\mathbf{v}}_{\mathrm{t}}$; (d) Construct the Doppler reduced-dimension transformation matrix $\mathbf{T}_{\mathrm{d}}=\mathbf{I}_{K} \otimes \tilde{\mathbf{w}}_{\mathrm{t}}(i) \otimes \tilde{\mathbf{w}}_{\mathrm{r}}(i) \in \mathbb{C}^{K M(N-J) \times K}$, and derive the Doppler reduced-dimension covariance matrix $\tilde{\mathbf{R}}_{\mathrm{d}}=\mathbf{T}_{\mathrm{d}}^{\mathrm{H}} \tilde{\mathbf{R}} \mathbf{T}_{\mathrm{d}}$ and the corresponding Doppler steering vector $\tilde{\mathbf{v}}_{\mathrm{d}}=\mathbf{T}_{\mathrm{d}}^{\mathrm{H}} \tilde{\mathbf{v}}\left(f_{\mathrm{s}, 0}, f_{\mathrm{d}, 0}\right)$. Then calculate the Doppler weight vector $\tilde{\mathbf{w}}_{\mathrm{d}}(i)=\tilde{\mathbf{R}}_{\mathrm{d}}^{-1} \tilde{\mathbf{v}}_{\mathrm{d}} / \tilde{\mathbf{v}}_{\mathrm{d}}^{\mathrm{H}} \tilde{\mathbf{R}}_{\mathrm{d}}^{-1} \tilde{\mathbf{v}}_{\mathrm{d}}$;

(e) Repeat performing (b), (c) and (d) to calculate $\tilde{\mathbf{w}}_{\mathrm{d}}(i), \quad \tilde{\mathbf{w}}_{\mathrm{t}}(i)$ and $\tilde{\mathbf{w}}_{\mathrm{r}}(i)$, respectively, until $\left\|\tilde{\mathbf{w}}_{\mathrm{d}}(i)-\tilde{\mathbf{w}}_{\mathrm{d}}(i-1)\right\| /\left\|\tilde{\mathbf{w}}_{\mathrm{d}}(i)\right\|<\varepsilon_{1} \quad\left(\quad 0<\varepsilon_{1} \ll 1 \quad\right) \quad$ and $\left\|\tilde{\mathbf{w}}_{\mathrm{t}}(i)-\tilde{\mathbf{w}}_{\mathrm{t}}(i-1)\right\| /\left\|\tilde{\mathbf{w}}_{\mathrm{t}}(i)\right\|<\varepsilon_{2}\left(0<\varepsilon_{2} \ll 1\right)$ (the tolerance thresholds $\varepsilon_{1}$ and $\varepsilon_{2}$ );

(f) In the end, the three low-dimension weight vectors $\tilde{\mathbf{w}}_{\mathrm{d}}, \tilde{\mathbf{w}}_{\mathrm{t}}$ and $\tilde{\mathbf{w}}_{\mathrm{r}}$ are resolved respectively based on tri-iterative optimization to synthesize the whole weight vector $\tilde{\mathbf{w}}=\tilde{\mathbf{w}}_{\mathrm{d}} \otimes \tilde{\mathbf{w}}_{\mathrm{t}} \otimes \tilde{\mathbf{w}}_{\mathrm{r}}$.

\subsection{Analysis of the Required Sample Number and the Computational Burden}

For the efficient estimation of $\tilde{\mathbf{R}}_{\mathrm{d}}, \tilde{\mathbf{R}}_{\mathrm{t}}$ and $\tilde{\mathbf{R}}_{\mathrm{r}}$ in our proposed MIMO-STAP method, the required sample number $\tilde{L}$ should be larger than or equal to $2 \max \{K, M,(N-J)\}$. Moreover, the computational complexity of the proposed method is merely $\mathrm{O}\left[I\left(\tilde{L} K^{2}+\tilde{L} M^{2}+\tilde{L}(N-J)^{2}\right)\right]+\mathrm{O}\left[I\left(K^{3}+M^{3}+(N-J)^{3}\right)\right]$ which involves the matrix estimation and inversion, where $I$ is the iteration number, and $I=4 \sim 5$ in the simulation when the iteration thresholds are set as $\varepsilon_{1}=\varepsilon_{2}=0.001$. Therefore, the sample requirement and computational load of the proposed MIMO-STAP method are far less than those of the full-dimension MIMO-STAP introduced in Sec. 2, which are $L \geq 2 K M N$ and $\mathrm{O}\left(L K^{2} M^{2} N^{2}\right)+\mathrm{O}\left(K^{3} M^{3} N^{3}\right)$.

Meanwhile, the required sample number $\hat{L}$ of the direct TRIA for clutter plus jamming suppression should be larger than or equal to $2 \max \{K, M, N\}$, and the computational complexity under the same iteration number is $\mathrm{O}\left[I\left(\hat{L} K^{2}+\hat{L} M^{2}+\hat{L} N^{2}\right)\right]+\mathrm{O}\left[I\left(K^{3}+M^{3}+N^{3}\right)\right]$. Since $N<K$ usually holds in general, the sample requirement of the proposed method approximates to that of the direct TRIA. However, the computational burden of the proposed method is further reduced, while the near clutter plus jamming suppression performance of MIMO-STAP can be achieved compared to the direct TRIA.

Under the typical parameters: temporal pulse number $K=16$, transmit array element number $M=10$, receive array element number $N=10$, jammer number $J=2$, and iteration number $I=5$, the corresponding quantitative comparisons based on the above analysis are listed in Tab. 1 to illustrate the advantage of our method in terms of the sample requirement and computational complexity. 


\begin{tabular}{|c|c|c|c|}
\hline & Full-dimension & Direct TRIA & $\begin{array}{c}\text { Proposed } \\
\text { method }\end{array}$ \\
\hline $\begin{array}{c}\text { Sample } \\
\text { requirement }\end{array}$ & $\geq 3200$ & $\geq 32$ & $\geq 32$ \\
\hline $\begin{array}{c}\text { Computational } \\
\text { complexity }\end{array}$ & $1.4653 \times 10^{6}$ & $1.0344 \times 10^{5}$ & $9.524 \times 10^{4}$ \\
\hline
\end{tabular}

Tab. 1. Quantitative comparisons of the sample requirement and computational complexity.

\section{Simulation Results}

In the simulation, the basic parameters for MIMOSTAP are $K=16, \quad M=10, N=10, \lambda=0.23 \mathrm{~m}, \quad d_{\mathrm{t}}=$ $=d_{\mathrm{r}}=0.115 \mathrm{~m}, \beta=1, T=500 \mu \mathrm{s}, V=115 \mathrm{~m} / \mathrm{s}, H=8000 \mathrm{~m}$, $N_{\mathrm{c}}=180$. Besides, the cone angle of the target is assumed to be located at the side-looking direction of the airborne radar, which means the normalized spatial frequency of the target is $f_{\mathrm{s}, 0}=0$. The tolerance thresholds in TRIA are set as $\varepsilon_{1}=\varepsilon_{2}=0.001$. The clutter-to-noise ratio (CNR) is $40 \mathrm{~dB}$.

Assume there exist two independent jammers at the jamming directions $\psi_{1}=60^{\circ}$ and $\psi_{2}=120^{\circ}$ in the scenario, i.e., $f_{\mathrm{sj}, 1}=0.25$ and $f_{\mathrm{sj}, 2}=-0.25$, respectively, and the jamming-to-noise ratios (JNRs) are both $40 \mathrm{~dB}$. Figure 3 pre-

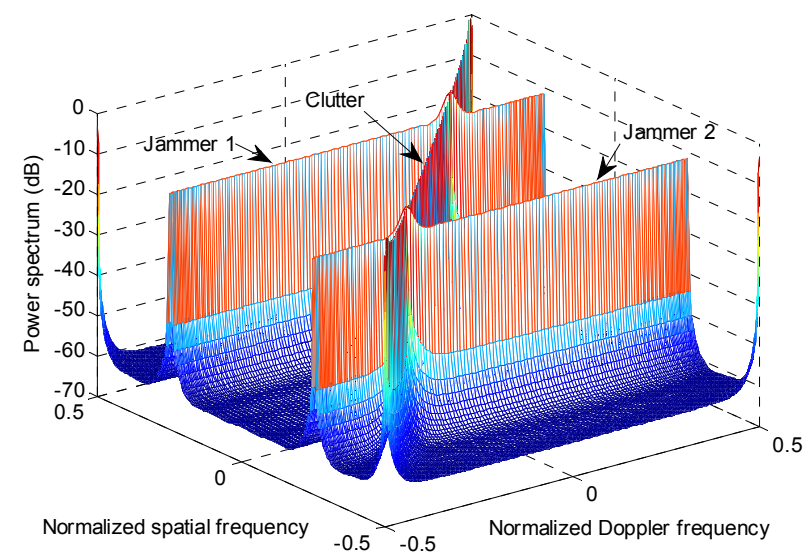

a)

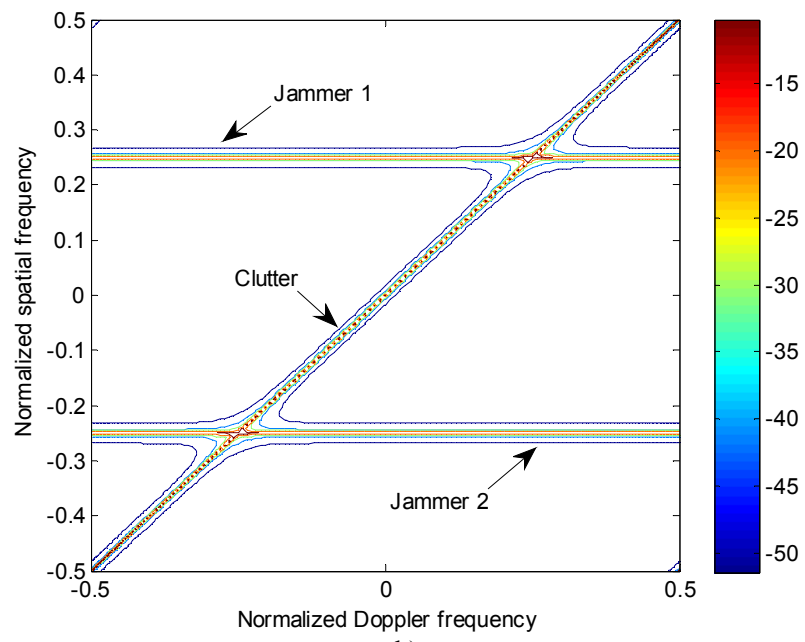

b)

Fig. 3. a) Clutter plus jamming spectrum. b) Two-dimensional spectrum projection. sents the corresponding clutter plus jamming spectrum and its projection. From Fig. 3, we can observe that the jamming spectrum is distributed along the Doppler frequencies and is discrete at spatial frequencies, which meets the analysis of the jamming signal model shown in Sec. 2.

The STAP performance is evaluated by improvement factor (IF), which is defined as the ratio of output signalto-clutter-plus-jamming-plus-noise ratio (SCJNR) to the input SCJNR. Then in the following simulations, the receive jamming plus noise sample number applied to estimate $\mathbf{R}_{\mathrm{r}}$ in the passive radar mode is fixed to 40 . As comparison, the optimum IF value is provided in each simulation figure indicated by "Optimum", which can be expressed as

$$
\begin{aligned}
& \mathrm{IF}_{\mathrm{opt}}=\frac{\mathrm{SCJNR}_{\mathrm{opt}}}{\mathrm{SCJNR}_{\mathrm{in}}} \\
& =\frac{\left|\mathbf{w}^{\mathrm{H}} \mathbf{v}\left(f_{\mathrm{s}, 0}, f_{\mathrm{d}, 0}\right)\right|^{2}(\mathrm{CNR}+\mathrm{JNR}+1) \sigma_{\mathrm{w}}^{2}}{\mathbf{w}^{\mathrm{H}} \mathbf{R} \mathbf{w}} \\
& =\mathbf{v}^{\mathrm{H}}\left(f_{\mathrm{s}, 0}, f_{\mathrm{d}, 0}\right) \mathbf{R}^{-1} \mathbf{v}\left(f_{\mathrm{s}, 0}, f_{\mathrm{d}, 0}\right)(\mathrm{CNR}+\mathrm{JNR}+1) \sigma_{\mathrm{w}}^{2}
\end{aligned}
$$

where the covariance matrix $\mathbf{R}=\mathbf{R}_{\mathrm{C}}+\mathbf{R}_{\mathrm{J}}+\mathbf{R}_{\mathrm{W}}$ is ideal and accurately known, not the estimated one produced by the training samples.

Figure 4 plots the IF against the iteration number in TRIA at the assumed normalized Doppler frequency of the target $f_{\mathrm{d}, 0}=0.25$. It is notable that $\tilde{L}=200$ training sample number is adopted in TRIA, which is obviously lower than the MIMO-STAP dimension $K M N$ and accords with the real clutter scenario. It can be seen from Fig. 4 that the proposed method converges to stability very well after 4 5 iterations, and the convergence value is approximate to that of the direct TRIA, which is about $3 \mathrm{~dB}$ lower than the ideal optimum IF value of the full-dimension MIMOSTAP. Moreover, the computational load is lower than that of the direct TRIA because of the receive dimension reduction in the jamming suppression stage.

Additionally, we consider the influence of different JNRs on the proposed method. Under the new JNR condi-

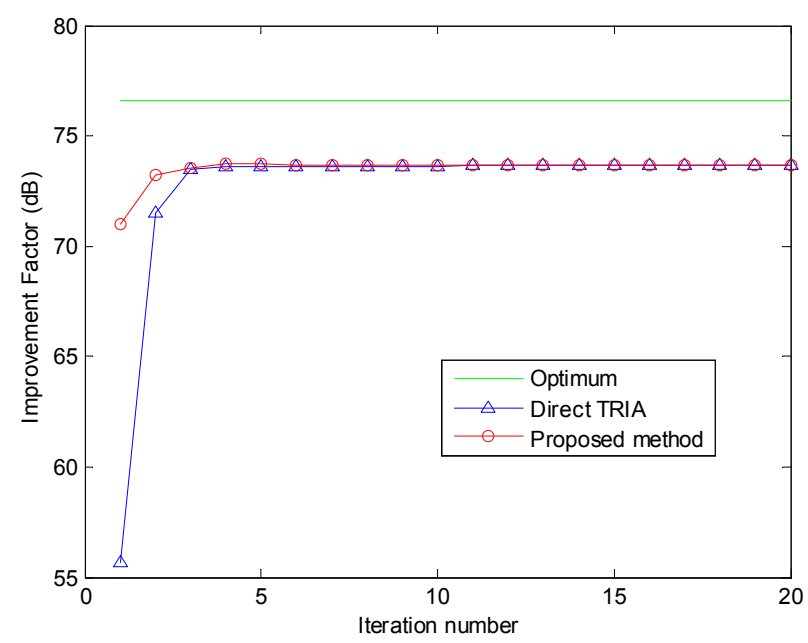

Fig. 4. IF versus the iteration number. 


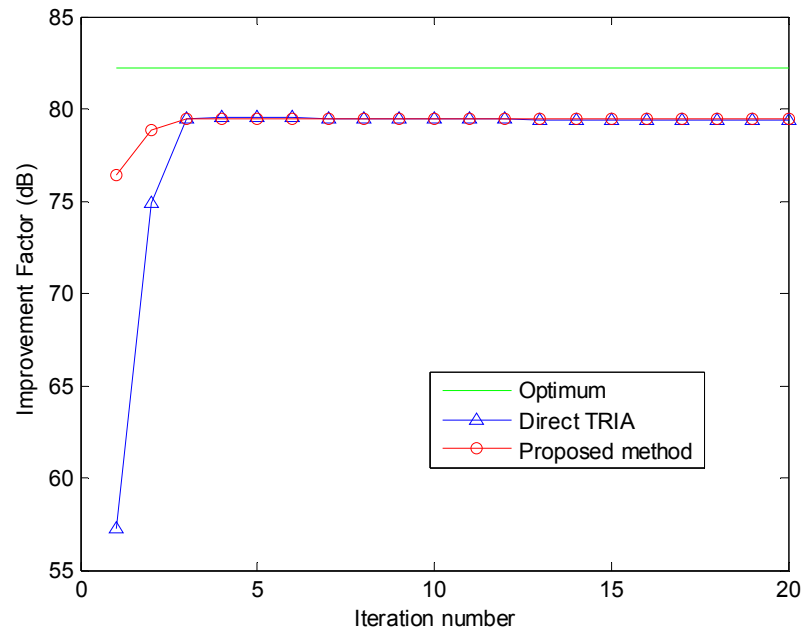

Fig. 5. IF versus the iteration number $\left(J N R_{1}=30 \mathrm{~dB}\right.$, $\left.J N R_{2}=50 \mathrm{~dB}\right)$

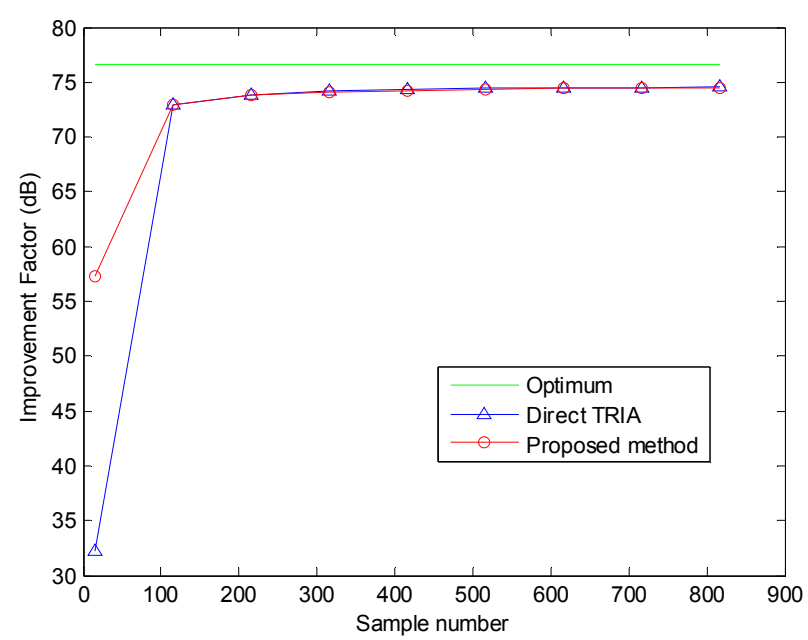

Fig. 6. IF versus the training sample number.

tion that $J N R_{1}=30 \mathrm{~dB}$ and $J N R_{2}=50 \mathrm{~dB}$, which is different from the previous simulation, the IF against the iteration number in TRIA at $f_{\mathrm{d}, 0}=0.25$ is depicted in Fig. 5. From Fig. 5, it can be found that the proposed method still owns stable convergence property in the changed JNR case, and only the convergence value is different from that in Fig. 4. Therefore, the theoretical correctness of our method is further validated. Afterwards, we return to the original JNR setting $\left(J N R_{1}=J N R_{2}=40 \mathrm{~dB}\right)$ to implement the following simulations.

Figure 6 presents the IF versus the number of training samples at $f_{\mathrm{d}, 0}=0.25$. Monte Carlo experiments are carried out 100 times to obtain the average values. The minimum sample number is taken as $\tilde{L}_{\min }=\max \{K, M, N\}=16$, for the feasibility of each low-dimension covariance matrix inversion in the direct TRIA. It can be observed that the proposed method converges fast with the training sample number, and exhibits superior IF performance than the direct TRIA under small sample number condition. In the practical non-homogeneous circumstance with insufficient IID samples, the proposed method owns advantage for anti-clutter and anti-jamming.

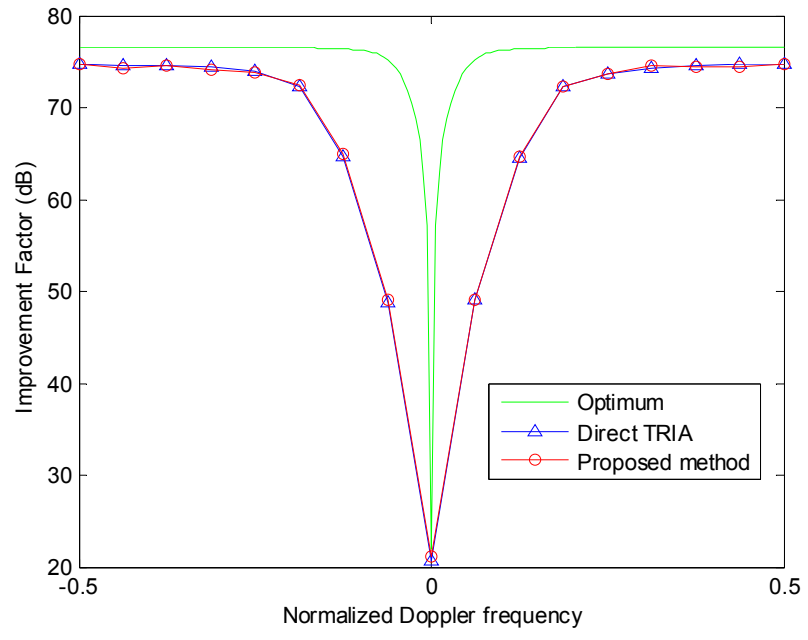

Fig. 7. IF versus normalized Doppler frequency.

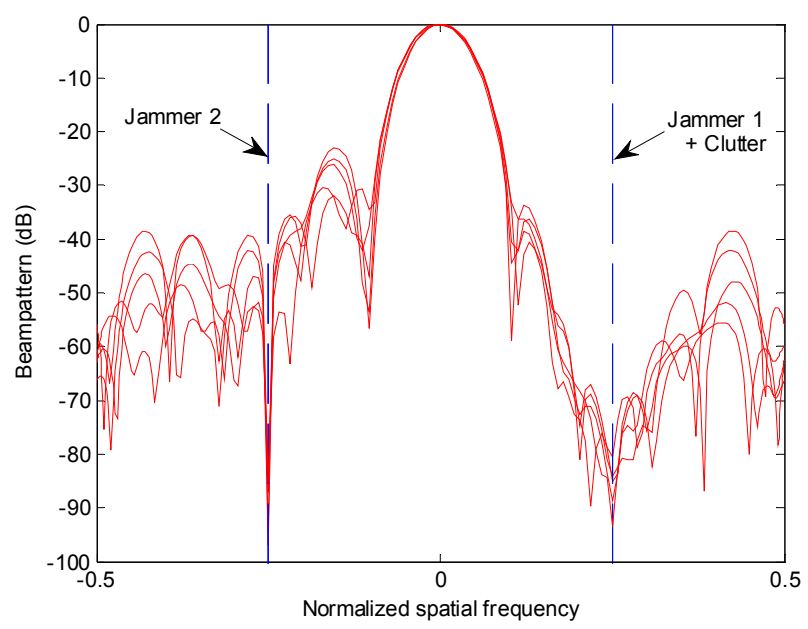

Fig. 8. Beampattern in the presence of jammers and clutter.

Figure 7 shows the IF performance against the Doppler frequency for the fixed $\tilde{L}=200$ samples. From Fig. 7, we can see that the IF curve of the proposed method resembles that of the direct TRIA versus different Doppler frequencies. This implies that the excellent minimum detectable velocity (MDV) performance can be achieved by the proposed cascaded MIMO-STAP method with lower computational cost, which is beneficial for the detection of the low-speed target in the clutter plus jamming background.

Figure 8 shows the spatial beampattern at $f_{\mathrm{d}, 0}=0.25$ in the presence of jammers and clutter of 5 independent trials by employing $\tilde{L}=200$ samples. We can find that the nullings are formed accurately in the jamming directions $f_{\mathrm{sj}, 1}=0.25$ and $f_{\mathrm{sj}, 2}=-0.25$, as well as the corresponding clutter notch, which further demonstrates the effectiveness of the proposed method.

\section{Conclusion}

Aiming at the typical jamming scenario, we propose a cascaded RD STAP method for airborne MIMO radar in this paper. Based on the different characteristics between 
jamming and clutter, we first cancel the jamming through utilizing the orthogonal property of its complementary subspace, with the reduction of the receive dimension. Next, the clutter is eliminated by TRIA based on the separation of the Doppler, transmit and receive weight vectors for MIMO-STAP. Simulation results demonstrate that the proposed method can provide excellent capability of clutter plus jamming suppression. Besides, the proposed cascaded MIMO-STAP method can effectively reduce the sample requirement and the computational burden, which is valuable for practical engineering application.

In our future research, the real scenario will be taken into consideration, including the clutter and the jammer. Meanwhile, more application tools can be utilized to execute our proposed method and produce practical results, such as field programmable gate array (FPGA) and C program, etc. Under the novel FPGA framework, the real-time requirement is a key factor when conducting the proposed cascaded MIMO-STAP algorithm. Thus, reasonable allocation and parallel implementation of the whole task are essential. On the other hand, efficient $\mathrm{C}$ program for realizing the conventional and fixed operations can be packaged into digital signal processor (DSP) and assembled with the new application system to further accelerate the processing speed.

\section{Acknowledgments}

This work was supported by the National Natural Science Foundation of China (61501513).

\section{References}

[1] FISHLER, E., HAIMOVICH, A., BLUM, R., et al. MIMO radar: an idea whose time has come. In Proceedings of the IEEE Radar Conference. Philadelphia (USA), 2004, p. 71-78. DOI: 10.1109/NRC.2004.1316398

[2] FiSHLER, E., HAIMOVICH, A. M., BLUM, R. S., et al. Spatial diversity in radars-models and detection performance. IEEE Transactions on Signal Processing, 2006, vol. 54, no. 3, p. 823 to 838. DOI: 10.1109/TSP.2005.862813

[3] HAIMOVICH, A. M., BLUM, R. S., CIMINI, L. J. MIMO radar with widely separated antennas. IEEE Signal Processing Magazine, 2008, vol. 25, no. 1, p. 116-129. DOI: 10.1109/MSP.2008.4408448

[4] LI, J., STOICA, P. MIMO radar with collocated antennas. IEEE Signal Processing Magazine, 2007, vol. 24, no. 5, p. 106-114. DOI: 10.1109/MSP.2007.904812

[5] BLISS, D.W., FORSYTHE, K.W. Multiple-input multiple-output (MIMO) radar and imaging: degrees of freedom and resolution. In Proceedings of the 37th Asilomar Conference on Signals, Systems and Computers. Pacific Grove (USA), 2003, p. 54-59. DOI: 10.1109/ACSSC.2003.1291865

[6] BLISS, D.W., FORSYTHE, K.W., DAVIS, S.K., et al. GMTI MIMO radar. In Proceedings of International Waveform Diversity and Design Conference. Kissimmee (USA), 2009, p. 118-122. DOI: 10.1109/WDDC.2009.4800327

[7] ZATMAN, M. The applicability of GMTI MIMO radar. In Proceedings of Asilomar Conference on Signals, System, and Computers. Pacific Grove (USA), 2010, p. 2138-2142. DOI: 10.1109/ACSSC.2010.5757928

[8] WARD, J. Space-Time Adaptive Processing for Airborne Radar. Technical Report 1015, Lexington (USA): MIT Lincoln Laboratory, 1994

[9] GUERCI, J. R. Space-Time Adaptive Processing for Radar. Norwood (USA): Artech House, 2003. ISBN: 1580533779

[10] KLEMM, R. Principles of Space-Time Adaptive Processing. London (UK): IEE, 2002. ISBN: 0863415660

[11] WU, Y., TANG, J., PENG, Y.N. Models and performance evaluation for multiple-input multiple-output space-time adaptive processing radar. IET Radar, Sonar and Navigation, 2009, vol. 3, no. 6, p. 569-582. DOI: 10.1049 /iet-rsn.2008.0025

[12] CHEN, C. Y., VAIDYANATHAN, P.P. MIMO radar space-time adaptive processing using prolate spheroidal wave functions. IEEE Transactions on Signal Processing, 2008, vol. 56, no. 2, p. 623 to 635. DOI: 10.1109/TSP.2007.907917

[13] ZHANG, W., HE, Z.S., LI, J., et al. Multistage multiple-beam beamspace reduced-dimension space-time adaptive processing for multiple-input-multiple-output radar based on maximum crosscorrelation energy. IET Radar, Sonar and Navigation, 2015, vol. 9, no. 7, p. 772-777. DOI: 10.1049/iet-rsn.2014.0226

[14] XIANG, C., FENG, D.Z., LV, H. Three-dimensional reduceddimension transformation for MIMO radar space-time adaptive processing. Signal Processing, 2011, vol. 91, no. 8, p. 2121-2126. DOI: $10.1016 /$ j.sigpro.2011.01.017

[15] HE, J., FENG, D.Z., MA, L. Reduced-dimension clutter suppression method for airborne multiple-input multiple-output radar based on three iterations. IET Radar, Sonar \& Navigation, 2015, vol. 9, no. 3, p. 249-254. DOI: 10.1049/iet-rsn.2014.0149

[16] REED, I., MALLETT, J., BRENNAN, L. Rapid convergence rate in adaptive arrays. IEEE Transactions on Aerospace and Electronic Systems, 1974, vol. 10, no. 6, p. 853-863. DOI: 10.1109/TAES.1974.307893

[17] KLEMM, R. Adaptive air- and spaceborne MTI under jamming conditions. In Proceedings of IEEE National Radar Conference. Boston (USA), 1993, p. 167-172. DOI: 10.1109/NRC.1993.270472

[18] RICHARDSON, P.G. STAP covariance matrix structure and its impact on clutter plus jamming suppression solutions. Electronics Letters, 2001, vol. 37, no. 2, p. 118-119. DOI: 10.1049/el:20010090

[19] HORN, R. A., JOHNSON, C. R. Matrix Analysis. New York (USA): Cambridge University Press, 1990. ISBN: 0-521-30586-1

\section{About the Authors ...}

Ting WANG was born in 1988. He is a Ph.D. candidate at Zhengzhou Institute of Information Science and Technology. He received his M.Sc. degree from Hefei Electronic Engineering Institute in 2013. His research interests include space-time adaptive processing and MIMO radar signal processing.

Yongjun ZHAO was born in 1964. He is a professor and Ph.D. advisor at Zhengzhou Institute of Information 
Science and Technology. He received his M.Sc. degree from Shanghai Jiaotong University in 1992 and his Ph.D. degree from Beijing Institute of Technology in 2008. His research interests include radar signal processing and array signal processing.

Shiwen CHEN was born in 1974. He is an associate professor at Zhengzhou Institute of Information Science and Technology. He received his M.Sc. degree and Ph.D. degree from Zhengzhou Institute of Information Science and
Technology in 2004 and 2013, respectively. His research interests include radar signal analyzing and processing.

Kunfan ZHANG was born in 1979. He is a lecturer at Zhengzhou Institute of Information Science and Technology. He received his M.Sc. degree and Ph.D. degree from Zhengzhou Institute of Information Science and Technology in 2003 and 2014, respectively. His research interests include radar system design and real-time signal processing. 